\title{
The blueberry component pterostilbene has potent anti-myeloma activity in bortezomib-resistant cells
}

\author{
GEGE CHEN $^{1 *}$, ZHIJIAN XU ${ }^{2 *}$, GAOMEI CHANG ${ }^{1 *}$, JUN HOU $^{1}$, LIANGNING HU ${ }^{1}$, YIWEN ZHANG ${ }^{1}$, \\ DANDAN YU ${ }^{1}$, BO LI $^{2}$, SHUAIKANG CHANG ${ }^{1}$, YONGSHENG XIE ${ }^{1}$, YONG ZHANG ${ }^{2}$, RONG WEI $^{1}$, \\ HUIQUN WU ${ }^{1}$, WENQIN XIAO ${ }^{1}$, XI SUN ${ }^{1}$, YI TAO $^{1}$, LU GAO $^{1}$, BOJIE DAI $^{3}$, \\ JUMEI SHI $^{1}$ and WEILIANG ZHU ${ }^{2}$ \\ ${ }^{1}$ Department of Hematology, Shanghai Tenth People's Hospital, Tongji University School of Medicine, \\ Shanghai 200072; ${ }^{2}$ CAS Key Laboratory of Receptor Research, Drug Discovery and Design Center, \\ Shanghai Institute of Materia Medica, Chinese Academy of Sciences, Shanghai 201203; \\ ${ }^{3}$ College of Life Science and Technology, Tongji University, Shanghai 200092, P.R. China
}

Received December 13, 2016; Accepted May 17, 2017

DOI: $10.3892 /$ or.2017.5675

\begin{abstract}
Multiple myeloma (MM) is an incurable hematologic malignancy because of its drug resistance. Pterostilbene (Pter) is found mainly in blueberries and grapes. The effects of Pter and its exact pharmacologic mechanisms on chemoresistant myeloma are not known. Herein, we investigated the anti-myeloma activity of Pter in bortezomib-resistant cell line H929R and explored the related mechanism of action for the first time. We found that Pter inhibited proliferation of H929R cells and promoted apoptosis of the cells through a caspase-dependent pathway, loss of mitochondrial membrane potential, and activation of Akt and p38 mitogen-activated protein kinase (MAPK) signaling pathways. DNA damage and S-phase arrest might be involved in Pter-related toxicity in H929R cells. Pter and the histone deacetylase inhibitors panobinostat or vorinostat inhibited proliferation of H929R cells in a synergistic manner. These data supported that Pter might be a promising natural compound for relapsed/refrac-
\end{abstract}

Correspondence to: Professor Weiliang Zhu, CAS Key Laboratory of Receptor Research, Drug Discovery and Design Center, Shanghai Institute of Materia Medica, Chinese Academy of Sciences, 555 Zuchongzhi Road, Shanghai 201203, P.R. China

E-mail:wlzhu@simm.ac.cn

Professor Jumei Shi, Department of Hematology, Shanghai Tenth People's Hospital, Tongji University School of Medicine, 301 Yanchang Road, Shanghai 200072, P.R. China

E-mail: shijumei@tongji.edu.cn

${ }^{*}$ Contributed equally

Key words: pterostilbene, multiple myeloma, bortezomib-resistant cells, apoptosis, cell cycle, histone deacetylase inhibitors tory myeloma therapy, especially against myeloma resistant to bortezomib chemotherapy.

\section{Introduction}

Multiple myeloma (MM) is a malignant disorder of clonal plasma cells. It accounts for $1 \%$ of all cancers diagnosed and $>10 \%$ of hematological malignancies (1). Advances in therapeutic strategies have resulted in improvements in the median survival of MM patients over the past decade from 3-4 years to 7-8 years (2). However, MM remains incurable due to its inevitable recurrence and progression, in which drug resistance remains a major problem $(3,4)$.

In recent years, scholars and the general public have been paying increasing attention to resveratrol. The latter can 'sensitize' resistant cells to chemotherapeutic agents by overcoming one or more mechanisms of chemoresistance (5). Various drug-resistant tumors, including lung carcinoma, pancreatic cancer, acute myeloid leukemia, promyelocytic leukemia, and MM, can be sensitized by resveratrol (6-9). Pterostilbene (3,5-dimethoxy-40-hydroxystilbene; Pter) is found mainly in blueberries, grapes and tree wood (10). Pter is a natural dimethylated analog of resveratrol but is superior to the latter with regard to liposolubility and bioavailability, which potentially makes it a more potent anticarcinogenic compound than resveratrol $(10,11)$. Pter has been demonstrated to execute apoptosis in tumors of the bladder, breast, colon, lung, pancreas, and stomach, as well as against leukemia cells (11). In bladder cancers, Pter has been found to inhibit the growth of sensitive and chemoresistant cancer cells by inducing cell cycle arrest, autophagy and apoptosis (12). Hence, Pter could be a new and promising agent for the treatment of chemoresistant cancer cells in the bladder. Pter and 3'-hydroxypterostilbene have been proved to be effective apoptosis-inducing agents in multiple-drug resistant (MDR) and BCR-ABL-expressing leukemia cells, suggesting its important role in the treatment of resistant hematologic malignancies (13). However, the effects of Pter and its exact pharmacologic mechanisms on 
other drug-resistant hematologic malignancies (especially in chemoresistant MM) are not known.

Apoptosis is a programmed form of cell death with typical morphologic features including cell shrinkage, chromatin condensation, DNA hydrolysis, nuclear fragmentation, and formation of apoptotic bodies $(14,15)$. The process is triggered by two principle mechanisms: the death receptor-mediated (extrinsic) pathway when death receptors are actived by bonding to corresponding death ligands or the mitochondrial (intrinsic) pathway initiated through release of mitochondrial intermembrane space proteins (15). Both pathways converge to activate a series of cysteine-dependent aspartate-specific proteases called caspases which are generally divided into two groups: the initiator caspases (caspase-2, -8, -9, and -10) and the effector caspases (caspase-3, -6, and -7) (16,17). Caspase-8, a key initiator caspase, could be actived by death ligands and receptors (Fas/FasL) via the extrinsic pathway, which is the first step of caspase cascade $(16,17)$. Caspase-3, one of the most important effector caspases, is a protease system that directly leads to the disintegration of apoptotic cells and is the center in the regulation of apoptosis $(16,17)$. Cleavage of caspase-3 triggered the inactivation of poly(ADP)ribose polymerase (PARP) and DNA fragmentation which are the hallmarks of apoptosis $(16,17)$. In general, the initiator caspases are usually autoactivated by particular pro-apoptotic stimuli while the effector caspases could be activated after proteolytic cleavage by the initiator caspases and then cause the cleavage or degradation of various specific substrates, leading ultimately to cell apoptosis (15-17).

Herein, we investigated the antiproliferative and proapoptotic effects of Pter on bortezomib-resistant H929R cells and explored the related mechanism of action. We found that Pter inhibited proliferation and induced caspase-dependent apoptosis as well as S-phase arrest of H929R cells. Loss of mitochondrial membrane potential (MMP) in Pter-treated cells was examined by flow cytometry. Moreover, downregulation of expression of phosphorylated Akt and upregulation of expression of phosphorylated p38 MAPK were observed by western blotting. Significantly, synergism between Pter and the histone deacetylase inhibitors (HDACIs) panobinostat or vorinostat resulted in toxicity to H929R cells. Thus, our work in the present study supported that Pter might be a promising natural compound for relapsed/refractory myeloma therapy, especially against myeloma resistant to bortezomib chemotherapy.

\section{Materials and methods}

Cells and cell culture. The human MM line H929 was purchased from American Type Culture Collection (Manassas, VA, USA). The human bortezomib-resistant MM line H929R was a kind gift from Professor Jian Hou (Department of Hematology, Changzheng Hospital, The Second Military Medical University, Shanghai, China). Human peripheral blood mononuclear cells (PBMCs) were separated by FicollHypaque density gradient centrifugation. Primary CD138 ${ }^{+}$ MM cells were obtained from the bone marrow of $\mathrm{MM}$ patients relapsed on bortezomib chemotherapy using magnetic bead selection (Miltenyi Biotech, Auburn, CA, USA). Peripheral blood samples and bone marrow samples were obtained from patients or healthy donors following acquisition of the study participants' written informed consent. The study was approved by the Shanghai Tenth People's Hospital Institutional Review Board. Bortezomib-resistant cell line H929R was cultured in the presence of $50 \mathrm{nM}$ bortezomib. All the cells mentioned above were grown in suspension in RPMI-1640 medium (Thermo Fisher Scientific, Waltham, MA, USA) supplemented with $10 \%$ fetal bovine serum and $1 \%$ penicillin streptomycin-glutamine, and incubated at $37^{\circ} \mathrm{C}$ in an atmosphere of $5 \%$ carbon dioxide.

Reagents and antibodies. Pter (purity, 98\%) was purchased from J\&K Chemicals (Shanghai, China). A stock solution of Pter $(100 \mathrm{mM})$ was dissolved in dimethyl-sulfoxide (DMSO; Sigma-Aldrich, St. Louis, MO, USA) and stored in the dark at $-20^{\circ} \mathrm{C}$. Antibodies against p38 MAPK (\#9212), Phospho-p38 MAPK (Thr180/Tyr182, \#9211), P13 Kinase p85 (19H8) (\#4257), Akt (\#9272), Phospho-Akt (Thr308, \#9275), Phosphate-Akt (Ser473, \#9271), SAPK/JNK (\#9252), phosphate-SAPK/JNK (Thr183/Tyr185, \#9251), Phospho-Histone H2A.X (Ser139, \#9718), Phospho-Chk1 (Ser296, \#2349), cdc25A (\#3652), CDK2 (\#2546), cleaved caspase-3 (Asp175, \#9661), cleaved caspase-8 (\#9496), PARP (\#9532) and $\beta$-actin (\#3700) were purchased from Cell Signaling Technology (Beverly, MA, USA). Antibodies against CyclinA2 (\#1547) were purchased from Epitomic (Burlingame, CA, USA). Fluorescence-conjugated secondary antibodies were purchased from Cell Signaling Technology. Cell Counting Kit-8 (CCK-8) was purchased from Dojindo (Mashikimachi, Japan). A MMP assay kit with JC-1 dye was purchased from Beyotime Institute of Biotechnology (Haimen, China).

Assays to measure cell proliferation and cytotoxicity. CCK-8 assays were undertaken to assess the viability of cancer cells with increasing concentrations of Pter or bortezomib. Cells $\left(4 \times 10^{5}\right.$ cells $\left./ \mathrm{ml}\right)$ were seeded in 96 -well plates with different concentrations of drugs for 24,48 , or $72 \mathrm{~h}$. Then, CCK-8 solution $(10 \mu \mathrm{l} /$ well $)$ was added to each well and inculcated for a further $2 \mathrm{~h}$ at $37^{\circ} \mathrm{C}$ in an atmosphere of $5 \% \mathrm{CO}_{2}$. Finally, absorbance was measured at $450 \mathrm{~nm}$ using a microplate reader.

Apoptosis assays. Cells ( $4 \times 10^{5}$ cells/ml) were seeded in 6-well plates with different concentrations of Pter for $48 \mathrm{~h}$ or with $30 \mu \mathrm{M}$ Pter for different time points. Then, cells were stained with Annexin V/propidium iodide (PI) (BD Pharmingen, Franklin Lakes, NJ, USA) and analyzed by flow cytometry following manufacturer instructions. Early (Annexin $\left.\mathrm{V}^{+}, \mathrm{PI}^{-}\right)$ and late (Annexin $\mathrm{V}^{+}, \mathrm{PI}^{+}$) apoptotic cells were counted.

TUNEL (terminal deoxynucleotide transferase dUTP nick-end labeling)/DAPI (4,6-diamidino-2-phenylindole) double-staining assay. TUNEL/DAPI double-staining was used to detect the apoptotic effect of Pter on the morphology of H929R cells. Cells $\left(4 \times 10^{5}\right.$ cells $\left./ \mathrm{ml}\right)$ were seeded in 6 -well plates with different concentrations of Pter for $24 \mathrm{~h}$. Then, the suspension was removed, and cells were fixed with $4 \%$ paraformaldehyde (Sigma-Aldrich) for $25 \mathrm{~min}$. Samples were examined by TUNEL assay according to the manufacturer's protocol (Promega Corp., Madison, WI, USA). Finally, DAPI (dilution 1:50,000; Sigma) was used to stain the nuclei. After 
treatment, cell apoptosis were analyzed using a fluorescence microscope (Zeiss Axiovert 25; Carl Zeiss, Jena, Germany).

Mitochondrial transmembrane potential assay. To determine whether the apoptosis induced by Pter was associated with activation of the mitochondrial apoptotic pathway, changes of mitochondrial transmembrane potential (MMP) in the apoptotic process were examined by flow cytometry using an MMP assay kit with JC-1 dye. Cells $\left(4 \times 10^{5}\right.$ cells $\left./ \mathrm{ml}\right)$ were cultured with different concentrations of Pter for $24 \mathrm{~h}$. Then, cells were washed with ice-cold phosphate-buffered saline (PBS) and incubated with $0.5 \mathrm{ml} \mathrm{JC}-1$ stain in a $37^{\circ} \mathrm{C}$ incubator for $20 \mathrm{~min}$. Finally, flow cytometry was carried out according to manufacturer's instructions.

Western blotting. Cells were treated with different concentrations of Pter and then lysed using lysis buffer $(100 \mathrm{mM}$ Tris-HCl, pH 6.8, 4\% sodium dodecyl sulfate, $20 \%$ glycerol). Equal amounts of proteins (30 $\mu \mathrm{g}$ per lane) were separated on $10 \%$ or $12.5 \%$ sodium dodecyl sulfate-polyacrylamide gels, transferred to nitrocellulose membranes, blocked with $5 \%$ skimmed milk or $5 \%$ bovine serum albumin for $1 \mathrm{~h}$, and incubated with primary antibodies $(1: 1,000)$ at $4^{\circ} \mathrm{C}$ overnight. Finally, membranes were treated with fluorescence-conjugated secondary antibodies $(1: 1,000)$ at room temperature for $60 \mathrm{~min}$ and detected by a two-color infrared laser imaging system (Odyssey; Li-Cor, Lincoln, NE, USA).

Statistical analyses. Data are the mean \pm standard deviation (SD). Statistical significance was determined by the Student's t-test or one-way ANOVA for multiple comparisons with SPSS v22.0 (IBM, Armonk, NY, USA). p $<0.05$ was considered significant. All experiments were carried out at least thrice.

\section{Results}

Pter inhibits proliferation of $H 929 R$ cells in a dose-dependent manner. H929R cells were obtained by increasing extracellular concentrations of bortezomib stepwise over 8 months (18). To verify the resistance in H929R cells, H929 and H929R cells were exposed to different concentrations of bortezomib for $48 \mathrm{~h}$ and the half-maximal inhibitory concentration $\left(\mathrm{IC}_{50}\right)$ was confirmed by CCK8 assays. Bortezomib inhibited proliferation of $\mathrm{H} 929$ cells effectively $\left(\mathrm{IC}_{50}=13.5 \pm 2.38 \mathrm{nM}\right.$ ), whereas H929R cells were more resistant to bortezomib $\left(\mathrm{IC}_{50}=143.2 \pm 4.34 \mathrm{nM}\right)$.

We first tested the antiproliferative activity of Pter in bortezomib-resistant H929R and drug-naive H929 cells. H929R and H929 cells were treated with 10, 20, 30, 40 and $50 \mu \mathrm{M}$ Pter for $48 \mathrm{~h}$ and the toxic effect of Pter in both cell lines was examined via CCK8 assays. We found that Pter significantly inhibited proliferation of H929R and H929 cells in a dose-dependent manner (Fig. 1A). To compare the antiproliferative effect of Pter on both cell lines, the $\mathrm{IC}_{50}$ was then calculated by CalcuSyn. The $\mathrm{IC}_{50}$ after $48 \mathrm{~h}$ of Pter treatment is $34.8 \pm 1.42$ and $22.83 \pm 1.13 \mu \mathrm{M}$ in $\mathrm{H} 929 \mathrm{R}$ and $\mathrm{H} 929$ cells, respectively. Compared with the growth-inhibitory effect of Pter on drug-naive H929 cells, the agent is also largely capable of inhibiting the proliferation of bortezomib-resistant cells, suggesting that Pter might be a promising natural compound
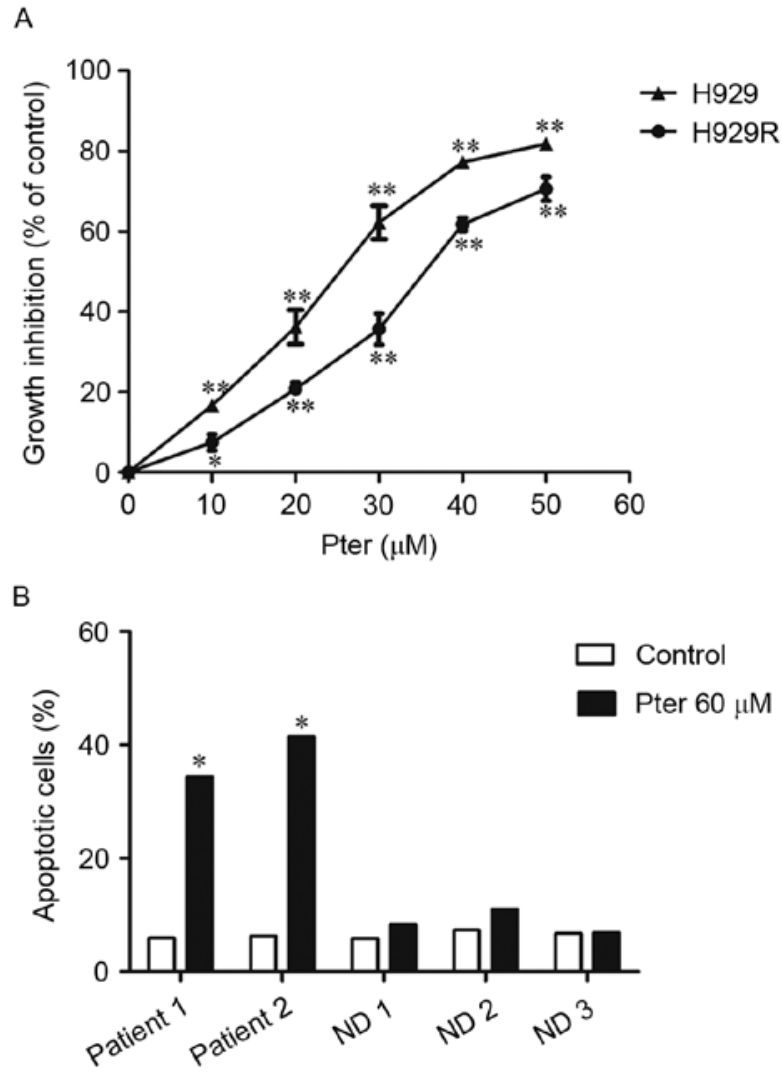

Figure 1. Pter inhibits proliferation of H929R cells in a dose-dependent manner. H929R and H929 cells were treated with 10, 20, 30, 40 and $50 \mu \mathrm{M}$ Pter for $48 \mathrm{~h}$ and the growth-inhibitory effect of Pter in both cell lines was examined via CCK8 assays (A). Primary CD $138^{+}$MM cells from two MM patients relapsed on bortezomib chemotherapy and PBMCs from three health donors were treated with medium or Pter $(60 \mu \mathrm{M}, 24 \mathrm{~h})$, stained with Annexin V/PI and analyzed via flow cytometry (B). ${ }^{*} \mathrm{p}<0.05,{ }^{* *} \mathrm{p}<0.01$, compared to the vehicle control group; Pter, pterostilbene; ND, normal donor; PBMCs, peripheral blood mononuclear cells.

for relapsed/refractory myeloma therapy, especially against myeloma resistant to bortezomib chemotherapy.

Herein, the effects and the related mechanisms of Pter in bortezomib-resistant myeloma were mainly explored. H929R cells were then treated with Pter for different times. However, time-dependent cytotoxicity was not observed within the given concentration range (data not shown). Additionally, cytotoxicity was also observed (by Annexin V/PI double-staining using flow cytometric analyses) in primary CD $138^{+}$cells isolated from MM patients relapsed on bortezomib without any obvious effects on PBMCs when Pter $(60 \mu \mathrm{M}, 24 \mathrm{~h})$ was administered (Fig. 1B), suggesting that Pter might be a safe agent for treatment of MM.

Pter induces apoptosis of H929R cells in a dose-and time-dependent manner. Apoptosis is characterized by cell shrinkage, chromatin condensation, DNA hydrolysis, nuclear fragmentation, and formation of apoptotic bodies $(14,15)$. TUNEL/DAPI double-staining was first carried out to detect the apoptotic effect of Pter on the morphology of H929R cells. Compared with the control, Pter (20 or $40 \mu \mathrm{M}, 24 \mathrm{~h}$ ) induced a series of morphological changes due to apoptosis (Fig. 2).

To investigate further the effects of Pter on H929R cells, Annexin V/PI double-staining was assessed via flow cytometry. 

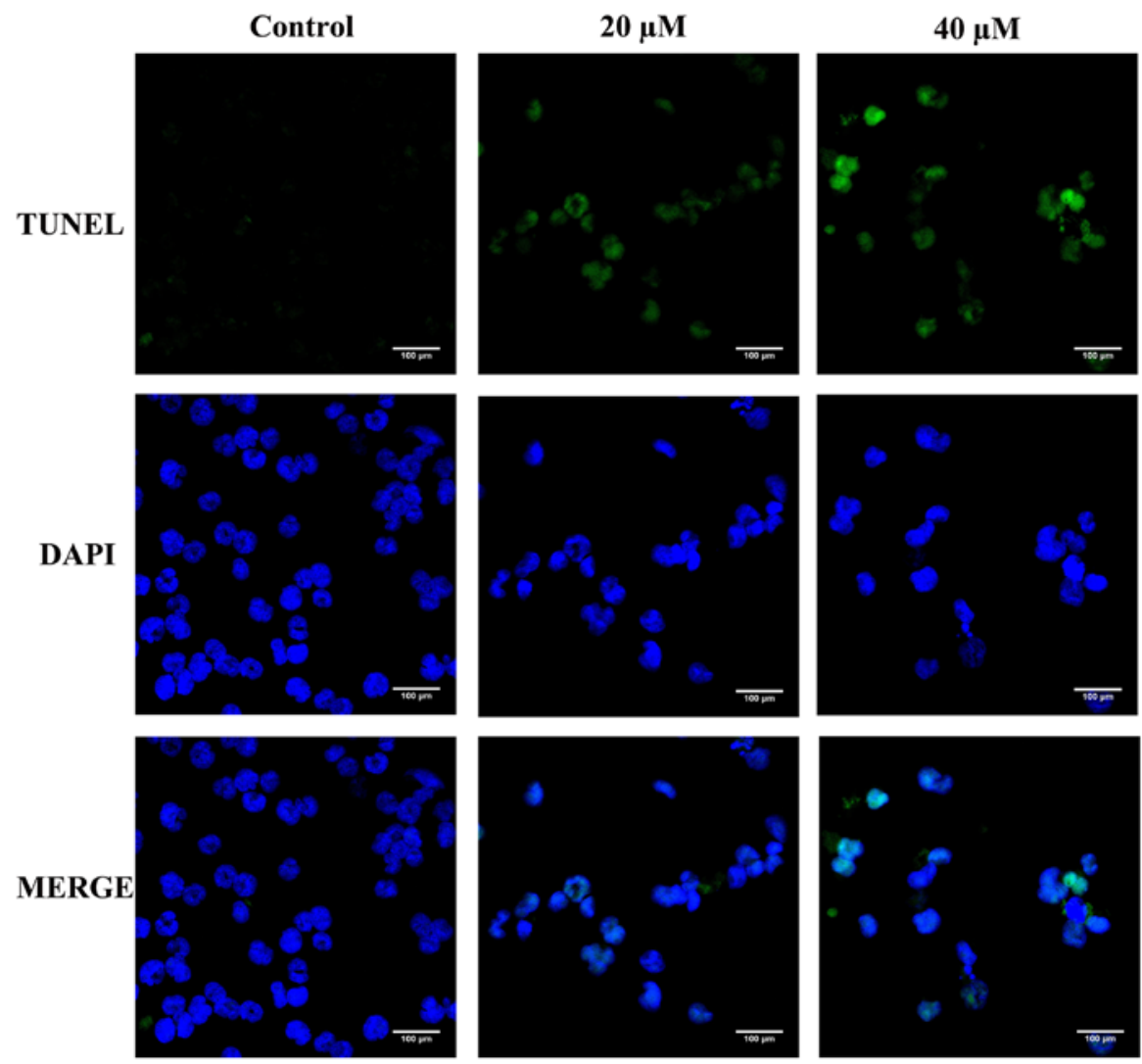

Figure 2. TUNEL (terminal deoxynucleotide transferase dUTP nick-end labeling)/DAPI (4,6-diamidino-2-phenylindole) double-staining of H929R cells. Representative images visualized by fluorescence microscopy after staining with TUNEL/DAPI. The green color in TUNEL staining denotes DNA fragmentation. DAPI staining was used for nuclear localization (magnification: $\mathrm{x} 400$, scale bar, $100 \mu \mathrm{m}$ ).

When cells were treated with different concentrations of Pter $(0,20$, or $40 \mu \mathrm{M})$ for $24 \mathrm{~h}$ or with $30 \mu \mathrm{M}$ Pter at different time points $(0,12,24$ or $48 \mathrm{~h})$, a significant apoptotic effect of Pter on H929R cells was noted (Fig. 3A and B). This phenomenon was associated with an increase in expression of caspase-3, caspase- 8 and poly(ADP)ribose polymerase (PARP) cleavage proteins (Fig. 3C).

Loss of mitochondrial transmembrane potential (MMP) is one of the key events in apoptosis focus on mitochondria $(19,20)$. Activation of the mitochondrial apoptotic pathway could be indirectly by determining whether the MMP collapses $(19,20)$. Next, changes in MMP in the apoptotic process were examined by flow cytometry using an MMP assay kit with JC-1 dye. A shift of red fluorescence in the control group to green fluorescence in drug-treated group was noted, showing that MMP levels in H929R cells treated with Pter (20 or $40 \mu \mathrm{M}, 24 \mathrm{~h}$ ) were much lower than those in the control group (Fig. 3D).

Studies have shown that the MAPK signaling pathway (especially c-Jun N-terminal kinase (JNK) and p38 MAPK) is essential for Pter-mediated activation of caspases (21). Another study has reported that a potent pan-PI3K/Akt inhibitor enhances the apoptotic effects of bortezomib in bortezomibresistant cells significantly, suggesting the roles of these signaling pathways in Pter-induced apoptosis (22). Therefore, we ascertained (by western blotting) if JNK, p38MAPK or $\mathrm{PI} 3 \mathrm{~K} / \mathrm{Akt}$ signaling pathways were activated in Pter-treated H929R cells. H929R cells were cultured with medium alone or Pter $(30 \mu \mathrm{M})$ for $48 \mathrm{~h}$, and then levels of PI3 kinase p85 (PI3K),
phospho-Akt (Thr308), phospho-Akt (Ser473), phosphorylation of p38 MAPK and JNK were measured. Downregulation of expression of PI3K, phosphorylated Akt and upregulation of expression of phosphorylated p38 MAPK were observed, but there were no changes in expression of phosphorylated JNK (Fig. 3E).

These findings suggested that Pter induced apoptosis of H929R cells in a dose-and time-dependent manner, and that this effect was associated with a caspase-dependent cell death pathway and loss of MMP. Also, Akt and p38 MAPK signaling pathways might be involved in Pter-treated H929R cells.

Pter triggers marked recruitment of $H 929 R$ cells in the $S$ phase of the cell cycle. To explore further the toxic effects of Pter on H929R cells, we next undertook cell cycle analyses on cells with medium alone or Pter using flow cytometry. After treatment of $\mathrm{H} 929 \mathrm{R}$ cells with Pter $(30 \mu \mathrm{M} ; 0,6,12$, and $24 \mathrm{~h}$ ), a remarkable accumulation of cells in the S phase was observed compared with that in the control ( $>1.5$-fold) (Fig. 4A and B). To investigate the molecular mechanisms, a series of proteins related to DNA damage and S-phase arrest was evaluated (using western blotting) in H929R cells treated with medium or Pter $(30 \mu \mathrm{M}, 24 \mathrm{~h})$. Levels of phospho-histone H2A.X, a sensitive marker for DNA double-strand breaks (DSBs) contributing to genomic instability and cancer treatment (23) and phospho-checkpoint kinase 1 (p-Chk1) proteins were upregulated significantly and proteins of cell division cycle 25 homologue A (cdc25A), cyclinA2 and cyclin-dependent kinase 2 (CDK2) were decreased notably in Pter-treated 

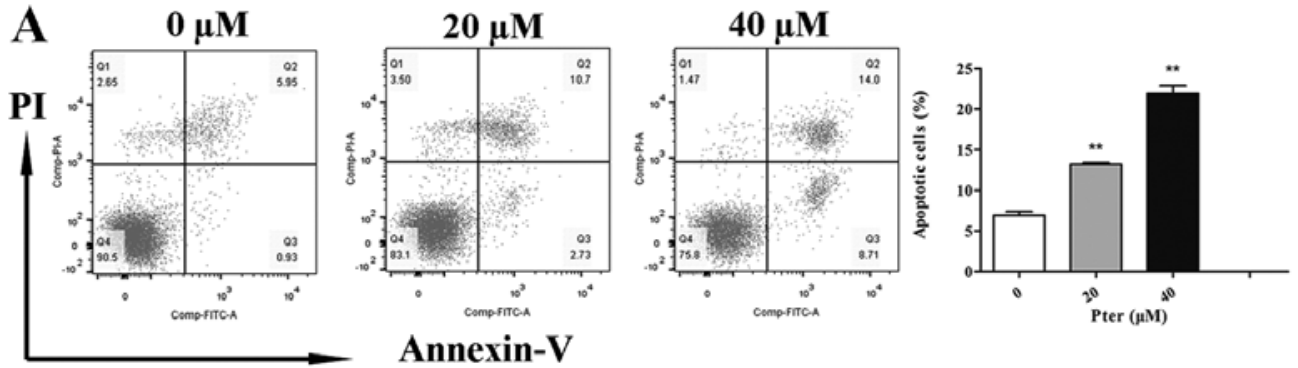

Annexin-V

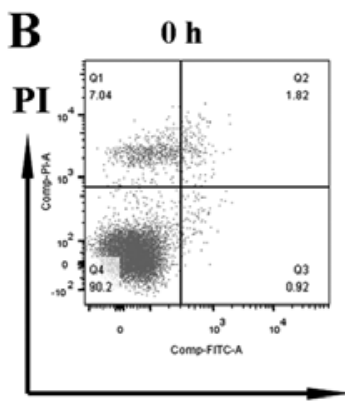

C

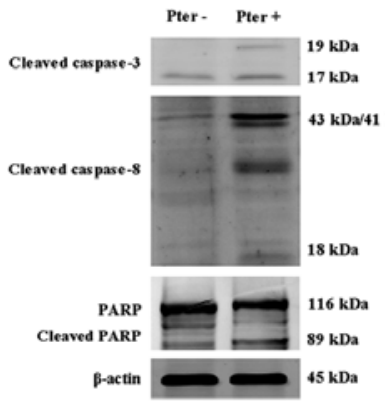

$\mathbf{E}$

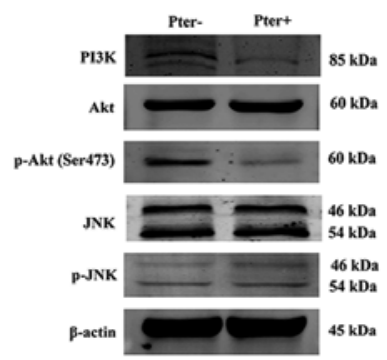

$12 \mathrm{~h}$

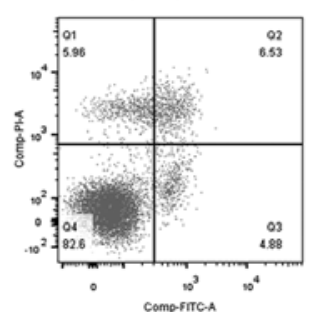

Annexin-V

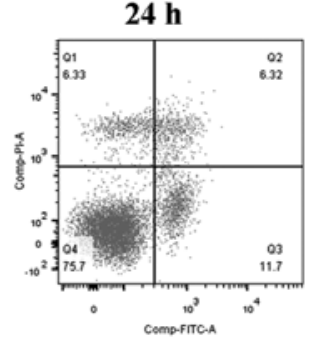

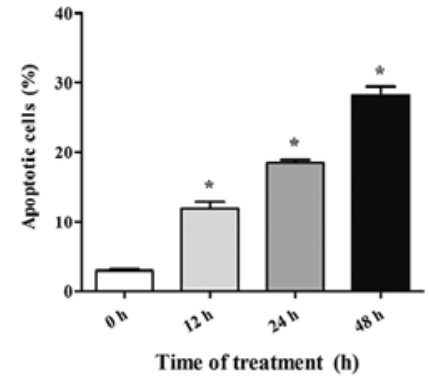

D

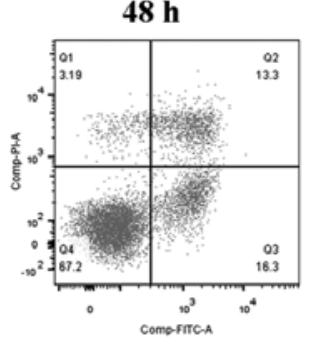

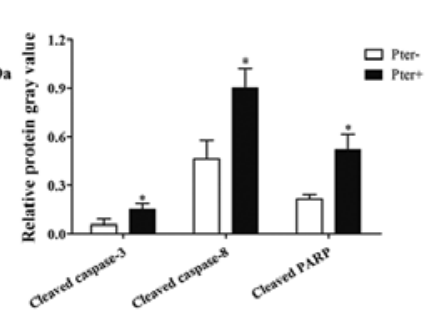
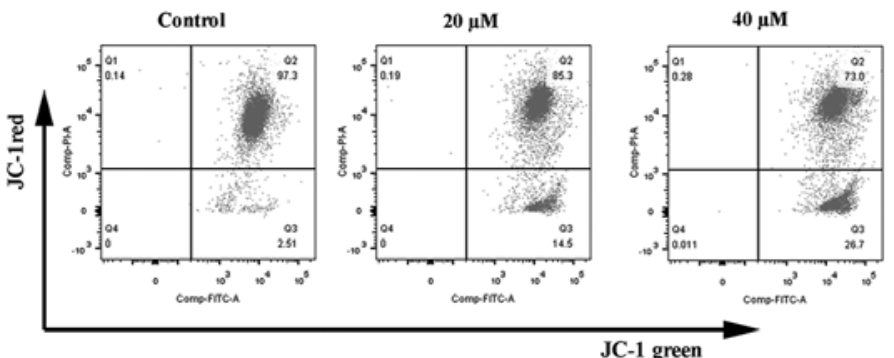

F
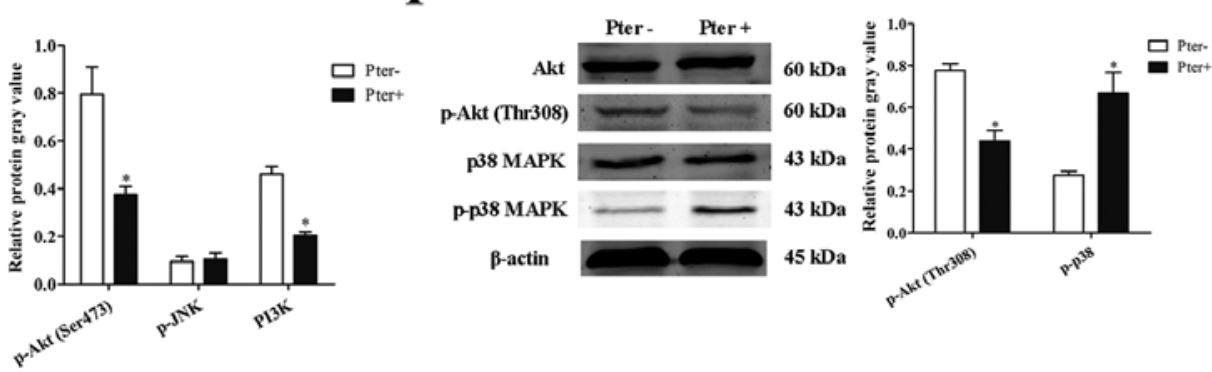

Figure 3. Pter induces apoptosis of H929R cells in a dose-and time-dependent manner. Quantitative analyses of apoptosis were done via Annexin V/propidium iodide (PI) double-staining using flow cytometry. H929R cells were treated with different concentrations of Pter for $24 \mathrm{~h}$ (A) or with $30 \mu \mathrm{M}$ Pter at different time points (B). H929R cells were treated with medium or Pter $(30 \mu \mathrm{M}, 48 \mathrm{~h})$. Western blotting for caspase-3, caspase-8, PARP cleaved proteins and quantification of relative cleaved protein expression (cleaved proteins gray values/ $\beta$-actin gray values) (C). Cells were treated with different concentrations of Pter for $24 \mathrm{~h}$ and changes in MMP were detected through JC-1 staining (D). Cells were treated with medium or Pter (30 $\mu \mathrm{M}, 48 \mathrm{~h}$ ). Western blotting for PI3 kinase p85 (PI3K), phospho-Akt (Ser473), phosphate-SAPK/JNK (p-JNK), phospho-Akt (Thr308), phospho-p38 MAPK (p-p38 MAPK) and quantification of relative proteins expression (phosphorylated proteins gray values/corresponding total proteins gray values, PI3K gray values/ $\beta$-actin gray values) (E). Data are the mean $\pm \mathrm{SD}$. ${ }^{*} \mathrm{p}<0.05,{ }^{* *} \mathrm{p}<0.01$ compared with the control group. Pter, pterostilbene.

groups (Fig. 4C). In conclusion, these findings suggested that DNA damage and S-phase arrest might be involved in Pterrelated toxicity in $\mathrm{H} 929 \mathrm{R}$.

Pter in combination with HDACIs results in cytoxic effect on H929R cells in a synergistic manner. Combination chemotherapy is one of the most effective strategies for patients with relapsed/refractory MM. To explore the potential of Pter for MM, we assessed the cytotoxic effects of Pter combined with HDACIs panobinostat or vorinostat. First, H929R cells were treated with different concentrations of panobinostat or vorinostat for $48 \mathrm{~h}$, and the $\mathrm{IC}_{50}$ values of the two drugs in $\mathrm{H} 929 \mathrm{R}$ cells were $32.5 \pm 0.91 \mathrm{nM}$ and $0.8 \pm 0.35 \mu \mathrm{M}$, respectively. Then, panobinostat $(25$ or $30 \mathrm{nM})$ or vorinostat $(0.6$ or $0.8 \mu \mathrm{M})$ was added to H929R cells treated with increasing concentrations of Pter $(0,10,20,30,40$, and $50 \mu \mathrm{M}, 48 \mathrm{~h})$ and the toxic effects on H929R cells were evaluated by CCK8 assays. The combination treatment with panobinostat $(25$ or $30 \mathrm{nM})$ or vorinostat ( 0.6 or $0.8 \mu \mathrm{M})$ and increasing concentrations of Pter sharply increased the growth inhibition of H929R cells (Fig. 5A and C). 

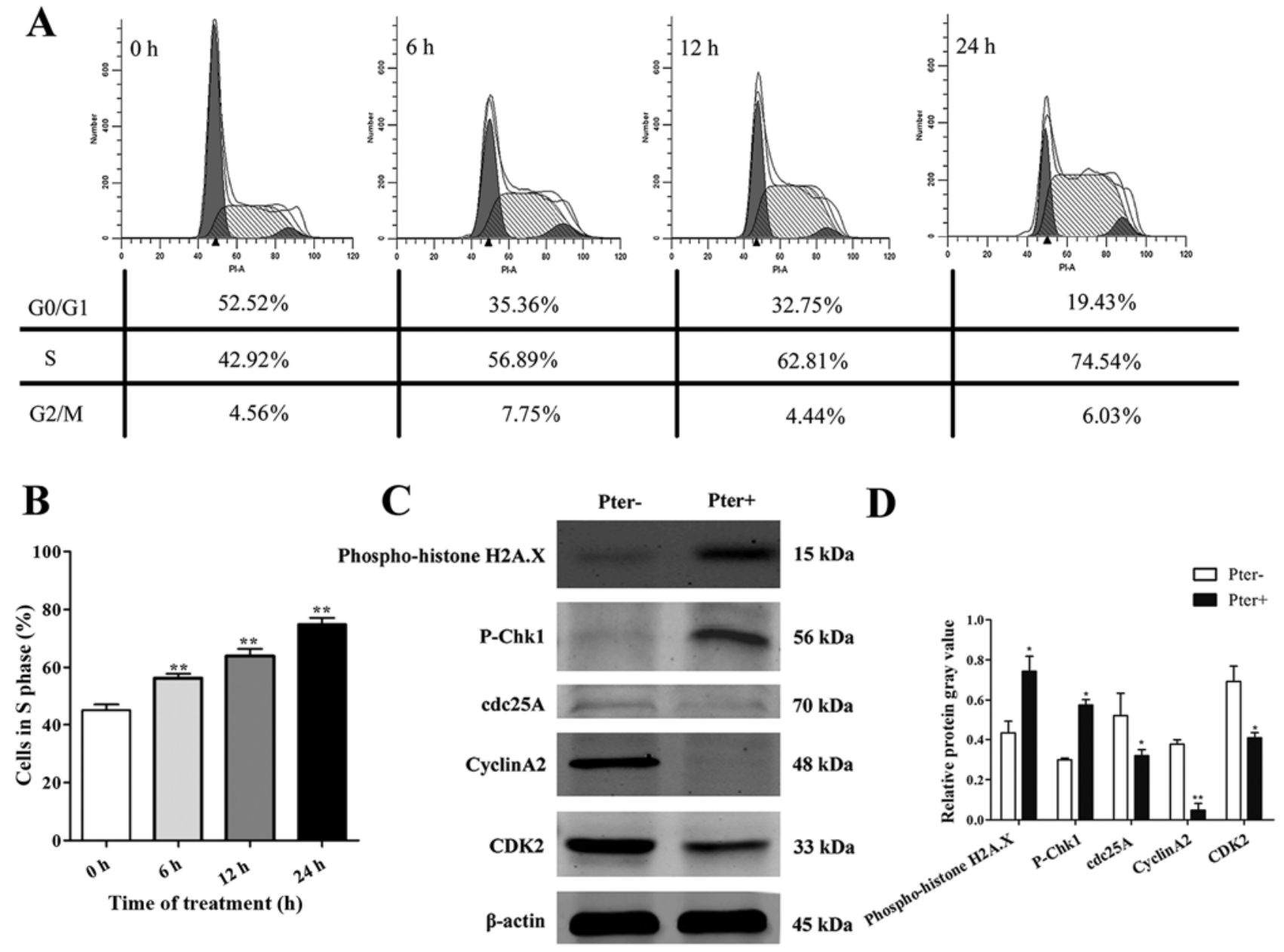

Figure 4. Pter triggers marked recruitment of H929R cells in the S phase of the cell cycle. Cell cycle analyses of H929R cells treated with medium alone or Pter $(30 \mu \mathrm{M})$ using flow cytometry. These data represented one of three experiments (A). The percentage of the S phase at $0,6,12$, and $24 \mathrm{~h}$ was $45.19 \pm 2.05 \%$, $56.27 \pm 1.51 \%, 63.88 \pm 2.48 \%$, and $74.9 \pm 2.16 \%$, respectively (B). Cells were treated with medium or Pter $(30 \mu \mathrm{M}, 24 \mathrm{~h})$. Western blotting for a series of proteins related to S-phase arrest and DNA damage. Quantification of relative protein expression (specific protein gray values/ $\beta$-actin gray values) (C). Data are the mean \pm SD. ${ }^{*} p<0.05,{ }^{* *} p<0.01$ compared with the control group. Pter, pterostilbene.

A synergistic effect was confirmed by combination index (CI) values using the Chou-Talalay method. As indicated in Fig. 5B and $\mathrm{D}$, median dose effect analyses showed the combination of Pter with HDACis, especially vorinostat induced synergistic cytotoxicity, with a CI $<1.0$ in H929R cells.

\section{Discussion}

As a first-generation proteasome inhibitor (PI), bortezomib is approved by the US Food and Drug Administration (FDA) for treatment of relapsed/refractory or newly diagnosed multiple myeloma (MM) (24). Bortezomib alone or in combination with other drugs (e.g. doxorubicin, melphalan, dexamethasone, immunomodulators) has considerable therapeutic clinical efficacy, but resistance and relapse are inevitable $(24,25)$. Until now, the mechanisms of bortezomib resistance have been understood incompletely $(3,25)$. Worse still, once MM patients have become refractory to bortezomib, poor outcome is inevitable, and median, overall survival, and event-free survival will be $<10$ months (4). Hence, the need to find strategies to overcome bortezomib resistance is urgent.

Resveratrol can sensitize cells resistant to chemotherapeutic agents by overcoming one or more mechanisms of chemore- sistance (5). In MM, resveratrol has been shown to enhance the apoptotic and anti-proliferative potential of bortezomib and thalidomide through downregulation of nuclear factor- $\kappa \mathrm{B}$ (NF- $\mathrm{kB}$ ), signal transducer and activator of transcription 3 (STAT-3) pathways (6). In another study, resveratrol was demonstrated to enhance the apoptotic potential of perifosine and bortezomib in drug-refractory MM and T-cell leukemia cells by enhancing recruitment of Fas/CD95 death receptors in the extrinsic pathway of apoptosis (26). Compared with resveratrol, Pter has similar pharmacologic benefits but exhibits much greater bioavailability (95\% vs. $20 \%$ ) and much longer half-life (105 vs. $14 \mathrm{~min}$ ), which makes it more potent for clinical use (10). Pter was found to inhibit growth of chemoresistant human bladder-cancer cells by inducing cell cycle arrest, autophagy and apoptosis (12). In docetaxel-induced MDR human lung cancer cell lines, Pter has been shown to inhibit cellular growth, cell cycle arrest, apoptosis and autophagy (27). In MDR leukemia cell lines, Pter was discovered to induce apoptosis through a caspase-independent mechanism, suggesting its utility in treatment of resistant hematologic malignancies (13). In addition, based on the anti-chemoresistant effects of Pter in other tumor types, we investigated the toxic effects of Pter in the bortezomib-resistant MM line H929R. 
A

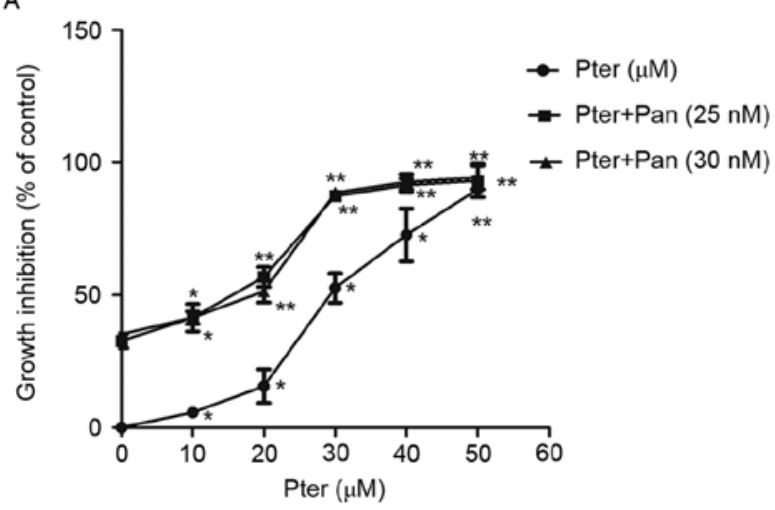

C

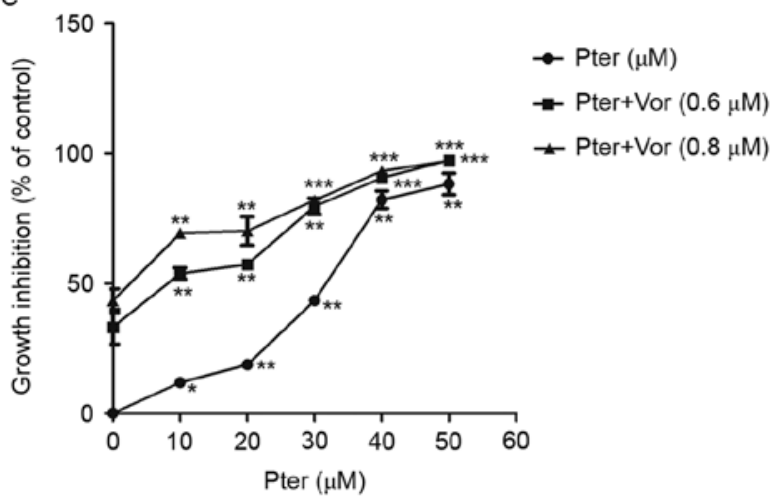

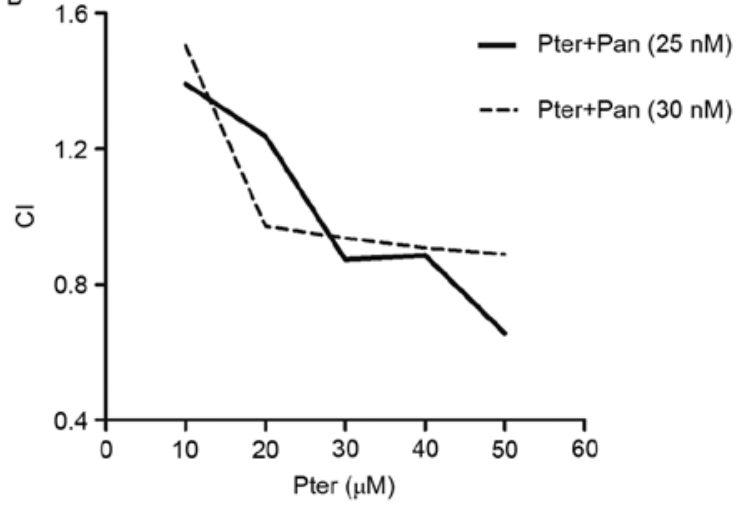

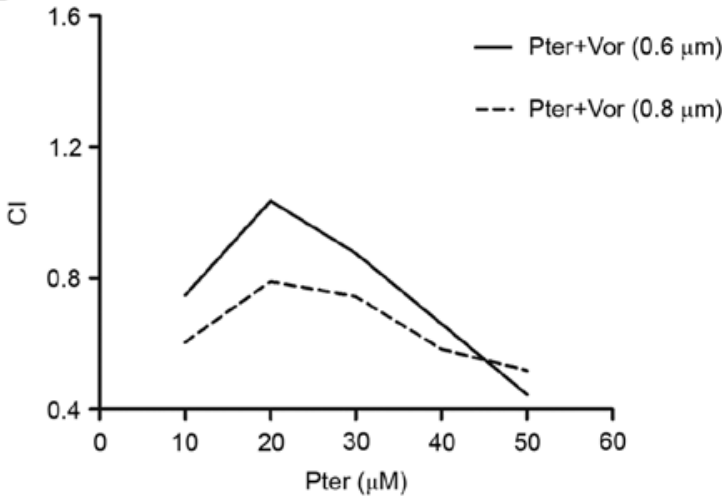

Figure 5. Pter and HDACIs result in toxic effects to H929R cells in a synergistic manner. Panobinostat or vorinostat was added to H929R cells treated with increasing concentrations of Pter, and the toxic effects on H929R cells evaluated by CCK-8 assays (A and C). CI values were calculated based on the medianeffect principle (B and D). CI $<1.0$ denotes synergistic interactions. Results are the mean values of three experiments. ${ }^{*}$ p $<0.05,{ }^{* *}$ p $<0.01,{ }^{* * *}$ p $<0.001$, compared to the vehicle control group; Pter, pterostilbene; Pan, panobinostat; Vor, vorinostat; CI, combination index.

Our data showed that Pter inhibited proliferation of H929R cells in a dose-dependent manner but time-dependent cytotoxicity was not observed. Of note, in MDR leukemia, Pter has been shown to induce dose-dependent inhibition of cell growth in all cell lines tested, but the time-dependent cytotoxicity was not mentioned (13). Studies have shown that Pter can induce a concentration- and time-dependent anti-proliferation effect in various tumor cell types, including melanoma, breast adenocarcinoma, and lung cancer (27-29); these findings are not entirely consistent with our findings. The difference in results may be associated with the types of cancer cells used in vitro studies. Therefore, further investigations on different myeloma cell lines in vitro and related animal models in vivo are needed.

In addition, our data suggested that Pter triggered apoptosis of H929R cells in a dose- and time-dependent manner, a result that is consistent with reports of apoptosis in melanoma after treatment with Pter and inositol hexaphosphate (29). TUNEL/DAPI double-staining was first done and we observed a series of morphologic changes due to apoptosis. Furthermore, the mechanisms involved in Pter-treated H929R cells were explored, and we found that both caspase-related proteins as well as MMP, p38 MAPK and Akt signaling pathways were associated with Pter-induced apoptosis. In human acute myeloid leukemia cell lines, Pter has been shown to be capable of inducing apoptosis through activation of caspase-8, -9 and -3, an MMP-dependent pathway, ERK1/2 and JNK, results that are consistent with our findings to a certain extent (13). Various studies have shown that activation of the PI3K/Akt signaling pathway often results in bortezomib resistance and MM recurrence $(22,30)$. In bortezomib-resistant cell lines (H929R, RPMI-8226R), a potent pan-PI3K inhibitor has been reported to downregulate phospho-Akt (p-Akt) expression markedly (22). In a previous study exploring the role of PI3K/Akt in the regulation of Azadirachtin-induced autophagy in SL-1 cells, Shao et al demonstrated decreased PI3K and phospho-Akt proteins in SL-1 cells following Azadirachtin treatment (31). Herein, we observed downregulation of PI3K, phospho-Akt (Ser473) and phospho-Akt (Thr308) proteins after exposure of H929R to Pter, suggesting that PI3K/Akt signaling pathway might be involved in Pter-induced apoptosis.

S-phase arrest is mediated by DNA damage activating Chk1/2, which inactivates cdc25A and downstream cyclinA2 and CDK2 (32). We found that Pter induced significant arrest of the S phase in H929R cells. Meanwhile, western blotting showed that expression of phospho-histone H2A.X, a sensitive marker for DNA DSBs that contributes to genomic instability and cancer treatment (23), and $\mathrm{p}-\mathrm{Chk} 1$ proteins were upregulated significantly and that expression of proteins of cdc25A, CDK 2 and cyclinA 2 were decreased considerably. These findings suggest that Pter could induce DNA damage and could result in S-phase arrest of H929R cells.

Mutations in apoptotic pathways, DNA damage, DNA repair or mitotic-checkpoint pathways can permit the survival or continued growth of cells with genomic abnormalities, thereby enhancing the likelihood of malignant 
transformation (33). Among them, the mitotic-checkpoint pathways and their associated proteins have critical roles in cell cycle arrest and cancer therapy (33-36). In general, the checkpoint pathway could check DNA damage and then forwards specific information to the protein cores of cell cycle machinery or replication apparatus, resulting in cell cycle arrest and DNA repair (34-36). However, if cellular damage cannot be properly repaired, checkpoint signaling pathway would convey information to apoptotic protein cores and upregulate proapoptotic-related proteins, leading to apoptosis $(35,36)$. Therefore, the anti-myeloma effects of Pter in H929R cells might be attributed to inhibition of MM cell proliferation and induction of cell apoptosis via mitotic-checkpoint signaling pathway.

Studies have reported that Pter combined with inositol6-phosphate (IP6) showed synergistic growth inhibition in melanoma (29). Herein, we report for the first time that Pter combined with an HDACI (panobinostat or vorinostat) could inhibit proliferation of H929R cells with CI values $<1$. As the first FDA-approved HDACI for patients with relapsed/ refractory $\mathrm{MM}$ who have undergone treatments previously (including bortezomib), panobinostat has been shown to be an effective agent in bortezomib-resistant myeloma cells and bortezomib-refractory MM patients $(37,38)$. Vorinostat (an oral non-selective class-I and class-II HDACI), has also been shown to be a potent anti-myeloma agent in preclinical and clinical studies $(39,40)$. Therefore, the synergistic effects of Pter and a HDACI (panobinostat or vorinostat) might be important for relapsed/refractory MM. This synergistic effect suggests that the serious side effects of HDACIs (panobinostat or vorinostat) could be reduced via combination with Pter. However, further validation and exploration of their cooperative effects and safety profiles are needed.

In conclusion, our findings suggest that the anti-myeloma activity of Pter in the bortezomib-resistant line H929R involves inhibition of cell proliferation, apoptosis induction, and S-phase arrest. Moreover, certain caspase-related proteins, loss of MMP, and activation of p38 MAPK and Akt signaling pathways are associated with Pter-induced apoptosis. Furthermore, our data first showed the synergistic effects of Pter in combination with HDACIs (panobinostat or vorinostat), which might be important for clinical trials of such combinations and related safety-profile studies.

\section{Acknowledgements}

This study was supported by grants from the National Natural Science Foundation of China (81372391, 81570190, 81529001, 81302699, 31271496, 81600174 and 81300443), and 'Personalized Medicines-Molecular Signature-based Drug Discovery and Development', Strategic Priority Research Program of the Chinese Academy of Sciences, grant no. XDA12020309.

\section{References}

1. Siegel RL, Miller KD and Jemal A: Cancer statistics, 2016. CA Cancer J Clin 66: 7-30, 2016.

2. Anderson KC: The 39th David A. Karnofsky Lecture: Bench-tobedside translation of targeted therapies in multiple myeloma. J Clin Oncol 30: 445-452, 2012.
3. Yang WC and Lin SF: Mechanisms of drug resistance in relapse and refractory multiple myeloma. BioMed Res Int 2015: 341430, 2015.

4. Kumar SK, Lee JH, Lahuerta JJ, Morgan G, Richardson PG, Crowley J, Haessler J, Feather J, Hoering A, Moreau P, et al; International Myeloma Working Group: Risk of progression and survival in multiple myeloma relapsing after therapy with IMiDs and bortezomib: A multicenter international myeloma working group study. Leukemia 26: 149-157, 2012.

5. Gupta SC, Kannappan R, Reuter S, Kim JH and Aggarwal BB: Chemosensitization of tumors by resveratrol. Ann NY Acad Sci 1215: 150-160, 2011

6. Bhardwaj A, Sethi G, Vadhan-Raj S, Bueso-Ramos C, Takada Y, Gaur U, Nair AS, Shishodia S and Aggarwal BB: Resveratrol inhibits proliferation, induces apoptosis, and overcomes chemoresistance through down-regulation of STAT3 and nuclear factor-kappaB-regulated antiapoptotic and cell survival gene products in human multiple myeloma cells. Blood 109: 2293-2302, 2007.

7. Zhu Y, He W, Gao X, Li B, Mei C, Xu R and Chen H: Resveratrol overcomes gefitinib resistance by increasing the intracellular gefitinib concentration and triggering apoptosis, autophagy and senescence in PC9/G NSCLC cells. Sci Rep 5: 17730, 2015.

8. Kato A, Naiki-Ito A, Nakazawa T, Hayashi K, Naitoh I, Miyabe K, Shimizu S, Kondo H, Nishi Y, Yoshida M, et al: Chemopreventive effect of resveratrol and apocynin on pancreatic carcinogenesis via modulation of nuclear phosphorylated GSK3 $\beta$ and ERK1/2. Oncotarget 6: 42963-42975, 2015.

9. Yaseen A, Chen S, Hock S, Rosato R, Dent P, Dai Y and Grant S: Resveratrol sensitizes acute myelogenous leukemia cells to histone deacetylase inhibitors through reactive oxygen speciesmediated activation of the extrinsic apoptotic pathway. Mol Pharmacol 82: 1030-1041, 2012.

10. Estrela JM, Ortega A, Mena S, Rodriguez ML and Asensi M: Pterostilbene: Biomedical applications. Crit Rev Clin Lab Sci 50: 65-78, 2013.

11. McCormack D and McFadden D: Pterostilbene and cancer: Current review. J Surg Res 173: e53-e61, 2012.

12. Chen RJ, Ho CT and Wang YJ: Pterostilbene induces autophagy and apoptosis in sensitive and chemoresistant human bladder cancer cells. Mol Nutr Food Res 54: 1819-1832, 2010.

13. Tolomeo M, Grimaudo S, Di Cristina A, Roberti M, Pizzirani D, Meli M, Dusonchet L, Gebbia N, Abbadessa V, Crosta L, et al: Pterostilbene and 3'-hydroxypterostilbene are effective apoptosis-inducing agents in MDR and BCR-ABL-expressing leukemia cells. Int J Biochem Cell Biol 37: 1709-1726, 2005.

14. Hollville E and Martin SJ: Measuring apoptosis by microscopy and flow cytometry. Curr Protoc Immunol 112: 1-24, 2016.

15. Koff JL, Ramachandiran S and Bernal-Mizrachi L: A time to kill: Targeting apoptosis in cancer. Int J Mol Sci 16: 2942-2955, 2015.

16. Shi Y: Mechanisms of caspase activation and inhibition during apoptosis. Mol Cell 9: 459-470, 2002.

17. Fan T-J, Han L-H, Cong RS and Liang J: Caspase family proteases and apoptosis. Acta Biochim Biophys Sin (Shanghai) 37: 719-727, 2005.

18. Zhu R, Xi H, Li YH, Jiang H, Zou JF and Hou J: Establishment of a bortezomib-resistant myeloma cell line and differential proteins analysis by MALDI-OF-MS. Zhejiang Da Xue Xue Bao Yi Xue Ban 38: 445-452, 2009 (In Chinese).

19. Green DR and Reed JC: Mitochondria and apoptosis. Science 281: 1309-1312, 1998.

20. Ni Chonghaile T, Sarosiek KA, Vo TT, Ryan JA, Tammareddi A, Moore VG, Deng J, Anderson KC, Richardson P, Tai YT, et al: Pretreatment mitochondrial priming correlates with clinical response to cytotoxic chemotherapy. Science 334: 1129-1133, 2011.

21. Hsiao PC, Chou YE, Tan P, Lee WJ, Yang SF, Chow JM, Chen HY, Lin CH, Lee LM and Chien MH: Pterostilbene simultaneously induced G0/G1-phase arrest and MAPK-mediated mitochondrial-derived apoptosis in human acute myeloid leukemia cell lines. PLoS One 9: e105342, 2014.

22. Yu W, Chen Y, Xiang R, Xu W, Wang Y, Tong J, Zhang N, Wu Y and Yan H: Novel phosphatidylinositol 3-kinase inhibitor BKM120 enhances the sensitivity of multiple myeloma to bortezomib and overcomes resistance. Leuk Lymphoma 58: 428-437, 2017.

23. Bonner WM, Redon CE, Dickey JS, Nakamura AJ, Sedelnikova OA, Solier S and Pommier Y: GammaH2AX and cancer. Nat Rev Cancer 8: 957-967, 2008. 
24. Chen D, Frezza M, Schmitt S, Kanwar J and Dou QP: Bortezomib as the first proteasome inhibitor anticancer drug: Current status and future perspectives. Curr Cancer Drug Targets 11: 239-253, 2011.

25. Murray MY, Auger MJ and Bowles KM: Overcoming bortezomib resistance in multiple myeloma. Biochem Soc Trans 42 : 804-808, 2014

26. Reis-Sobreiro M, Gajate $\mathrm{C}$ and Mollinedo F: Involvement of mitochondria and recruitment of Fas/CD95 signaling in lipid rafts in resveratrol-mediated antimyeloma and antileukemia actions. Oncogene 28: 3221-3234, 2009.

27. Hsieh MJ, Lin CW, Yang SF, Sheu GT, Yu YY, Chen MK and Chiou HL: A combination of pterostilbene with autophagy inhibitors exerts efficient apoptotic characteristics in both chemosensitive and chemoresistant lung cancer cells. Toxicol Sci 137: 65-75, 2014.

28. Yang Y, Yan X, Duan W, Yan J, Yi W, Liang Z, Wang N, Li Y, Chen W, Yu S, et al: Pterostilbene exerts antitumor activity via the Notch1 signaling pathway in human lung adenocarcinoma cells. PLoS One 8: e62652, 2013.

29. Schneider JG, Alosi JA, McDonald DE and McFadden DW: Effects of pterostilbene on melanoma alone and in synergy with inositol hexaphosphate. Am J Surg 198: 679-684, 2009.

30. Ikeda H, Hideshima T, Fulciniti M, Perrone G, Miura N, Yasui H, Okawa Y, Kiziltepe T, Santo L, Vallet S, et al: PI3K/p110\{delta\} is a novel therapeutic target in multiple myeloma. Blood 116 : $1460-1468,2010$

31. Shao X, Lai D, Zhang L and Xu H: Induction of autophagy and apoptosis via PI3K/AKT/TOR pathways by azadirachtin A in Spodoptera litura cells. Sci Rep 6: 35482, 2016.
32. Wahl DR and Lawrence TS: Integrating chemoradiation and molecularly targeted therapy. Adv Drug Deliv Rev 109: 74-83, 2017.

33. Kastan MB and Bartek J: Cell-cycle checkpoints and cancer. Nature 432: 316-323, 2004.

34. Golubnitschaja O: Cell cycle checkpoints: The role and evaluation for early diagnosis of senescence, cardiovascular, cancer, and neurodegenerative diseases. Amino Acids 32: 359-371, 2007.

35. Walworth NC: Cell-cycle checkpoint kinases: Checking in on the cell cycle. Curr Opin Cell Biol 12: 697-704, 2000.

36. Pietenpol JA and Stewart ZA: Cell cycle checkpoint signaling: Cell cycle arrest versus apoptosis. Toxicology 181-182: 475-481, 2002.

37. Raedler LA: Farydak (panobinostat): First HDAC inhibitor approved for patients with relapsed multiple myeloma. Am Health Drug Benefits 9: 84-87, 2016.

38. Corrales-Medina FF, Manton CA, Orlowski RZ and Chandra J: Efficacy of panobinostat and marizomib in acute myeloid leukemia and bortezomib-resistant models. Leuk Res 39: 371-379, 2015.

39. Maiso P, Carvajal-Vergara X, Ocio EM,López-Pérez R, Mateo G, Gutiérrez N, Atadja P, Pandiella A and San Miguel JF: The histone deacetylase inhibitor LBH589 is a potent antimyeloma agent that overcomes drug resistance. Cancer Res 66: 5781-5789, 2006.

40. Afifi S, Michael A, Azimi M, Rodriguez M, Lendvai N and Landgren O: Role of histone deacetylase inhibitors in relapsed refractory multiple myeloma: A focus on vorinostat and panobinostat. Pharmacotherapy 35: 1173-1188, 2015. 